\title{
Agenda institucional de la enseñanza media técnico-profesional en Chile, 2009-2018
}

\author{
Institucional agenda for vocational secondary education in Chile,
} 2009-2018

\section{Vanessa Orrego Tapia ${ }^{1}$}

\section{Resumen}

La enseñanza media técnico-profesional es un tema pendiente en la política pública chilena, pues, aunque en su agenda institucional se han discutido desafíos y propuestas a través de tres documentos oficiales publicados por el Ministerio de Educación, los avances concretos han sido escasos. En este escenario se desarrolla el siguiente ensayo, cuyo objetivo es revisar el discurso oficial sobre la educación media técnica profesional en la agenda institucional en Chile entre 2009-2018. El análisis se focaliza en las continuidades, como la priorización otorgada a la calidad educativa e institucionalidad, y las rupturas entre los documentos, particularmente aquella que acusa la mezcla de enfoques teóricos. Las conclusiones también relevan dos temáticas ausentes: la innovación educativa y el enfoque de género.

Palabras clave: Enseñanza media técnico-profesional; agenda institucional; Chile.

\begin{abstract}
Vocational secondary education is a pending issue in Chilean public policy, because, although challenges and proposals have been discussed in its

\footnotetext{
${ }^{1}$ Magíster en métodos de investigación social, Universidad Diego Portales y magíster en estudios latinoamericanos, Leiden University. Investigadora en Elige Educar, Centro de Políticas Públicas de la Pontificia Universidad Católica de Chile. Email: vanessa.orrego.tapia@gmail.com
} 
institutional agenda through three official documents published by the Ministry of Education, concrete progress has been scarce. In this scenario, the objective of the following essay is to review the official discourse on vocational secondary education on the institutional agenda in Chile between 2009-2018. The analysis focuses on continuities, such as the prioritization given to educational quality and institutionality, and the ruptures between documents, particularly the mixture of theoretical approaches. The conclusions also reveal two absent themes: educational innovation and the gender approach.

Keywords: Vocational secondary education; institutional agenda; Chile.

\section{Introducción}

Tal como en otros países, la formación técnico-profesional (FTP) en Chile es una modalidad que incentiva el aprendizaje teórico-práctico relevante para un campo ocupacional específico a través del desarrollo de aptitudes, competencias, habilidades y conocimientos (MINEDUC, 2012). La FTP se separa en dos momentos: la formación profesional y capacitación inicial (FPC-I) y la formación profesional y capacitación continua (FPC-C). La primera está dirigida a los jóvenes al inicio de sus trayectorias profesionales y previo al ingreso al mercado de trabajo, mientras la segunda comprende la actualización de competencias en los adultos. Así, la FCP-I ocurre en el nivel de la educación secundaria y superior; mientras la FCP-C es el aprendizaje abierto y para toda la vida (Sevilla, 2017). El presente ensayo se centra en el nivel inicial o FPC-I, particularmente en la educación media técnico-profesional (EMTP).

En Chile, la EMTP se ofrece en los dos últimos años de la educación media y en paralelo con la enseñanza media científico-humanista (EMCH) que se caracteriza por su orientación académica general. Desde el Decreto 452 de 2013, el cual aprobó sus últimas bases curriculares, la EMTP ofrece 35 especialidades y 17 menciones, agrupadas en 15 sectores económicos. Ahora bien, a pesar de esta distinción orientada a la formación diferenciada, la modalidad también debe asegurar contenidos mínimos de la formación general. Consecuentemente, la 
EMTP ofrece tanto un título de técnico en nivel medio, tras la finalización de una práctica laboral, como una licenciatura de enseñanza media, tal como la EMCH.

Comparado con otros niveles educativos, existe una suerte de silencio en sus políticas públicas. Aunque la insatisfacción con el nivel y la demanda ciudadana es conocida, no ha existido una reforma profunda desde hace un cuarto de siglo (Bellei et al., 2020), incluso en un escenario regional, recuerdan Llisterri et al. (2014), donde existe consenso sobre la urgencia de un cambio estructural en la producción económica hacia actividades de mayor valor agregado. Un cambio que requiere capital humano avanzado, a través de la educación media y superior, que sea capaz de absorber las innovaciones y llevar la competitividad de las empresas al mercado global. Por el contrario, en la EMTP en Chile es común encontrar la exportación de políticas educativas diseñadas para la educación básica (Bellei et al., 2020) o para todo el sistema escolar, obviando las especificidades del nivel, modalidad, estudiantes y profesores. Un clásico ejemplo de esto último está en el diseño de pruebas estandarizadas para alumnos y docentes, pues éstas tienden a privilegiar la enseñanza academicista y general (Arellano y Donoso, 2020; MINEDUC, 2012; UNESCO, 2015).

Ahora bien, a pesar de ello, en las últimas dos décadas han existido debates acerca de las principales debilidades del sistema y los caminos para avanzar hacia una EMTP de calidad y pertinente para el país, sus estudiantes y docentes, como se verá en los tres documentos que se revisaran. De esa manera, su agenda institucional no ha estado estática y ha definido objetivos, propuestas, prioridades, soluciones y metas a través del trabajo de diversas comisiones de expertos, instancias que han ayudado a contornear la definición oficial del problema público de la FTP. Una definición oficial que aquí se revisa.

En este contexto se enmarca el siguiente ensayo, cuyo objetivo es revisar el discurso oficial sobre la educación media técnica profesional en la agenda institucional en Chile entre 2009-2018. En particular se revisan tres documentos que, organizados y publicados por el Ministerio de Educación (MINEDUC), vienen a resumir y plasmar el contenido de la agenda institucional sobre la modalidad en los últimos años: Bases para una política de formación técnico-profesional en Chile 
(MINEDUC, 2009), Política nacional de formación técnico-profesional (MINEDUC, 2016) y Estrategia nacional de formación técnico-profesional (MINEDUC, 2018a). Los tres documentos simbolizan la definición oficial del problema público de la formación técnico profesional, en general, y la enseñanza media técnicoprofesional, en específico, en la agenda institucional de Chile.

El enfoque teórico propuesto para este ensayo es el construccionismo social, el cual entiende que la definición de los problemas públicos en la agenda institucional, gubernamental o formal es un proceso social que refleja las creencias, valores e interpretaciones que los actores tienen sobre condiciones que, en apariencia, son materiales y objetivas (Blumer, 1971; Elder y Cobb, 1993). Un proceso que, además, es transversal al desarrollo de toda política. Esta mirada se opone a los enfoques tradicionales, donde la definición de los problemas públicos es la primera fase del ciclo de construcción de la política pública (Aguilar, 1993; Weiss, 1989). Por el contrario, desde el construccionismo social, la definición de un problema público cubre, incluso, la etapa de implementación.

La tesis a defender en este ensayo destaca la construcción permanentemente en proceso del problema público de la FTP, particularmente la EMTP, como un discurso que en Chile no ha logrado ser uniforme. Así, junto a ciertas continuidades que atraviezan transversalmente los tres documentos discutidos, hay importantes rupturas. La más significativa discontinuidad es probablemente teórica y evidencia las discusiones de enfoque que subyacen en la formación técnico-profesional no sólo en Chile.

El ensayo se divide en dos apartados, además de la introducción y conclusión. En primer lugar, se presenta el contenido de la agenda gubernamental en la EMTP en Chile describiendo las distintas propuestas que existen en los tres documentos oficiales revisados. En segundo lugar, se analizan críticamente las continuidades y rupturas en el camino trazado por los mismos documentos.

\section{Políticas y estrategias que construyen agenda en el nuevo milenio}

Tras un intenso período de discusiones para la reforma educacional de 1998 (Castro y Orellana, 2010; MINEDUC, 2012; Sepúlveda, 2017; 2019) la agenda 
institucional de la FTP estuvo en pausa. Si bien una parte puede explicarse por la puesta en práctica de algunas de las modificaciones fijadas en la reforma, incluyendo un plan masivo de formación para implementar los cambios fijados (Miranda, 2003), parece evidente que la temática perdido prioridad en la agenda educativa. Entre 2000-2010 se formaron varias comisiones para abordar diversos desafíos educativos en el país (MINEDUC, 2009; 2016; 2018a), pero ninguna posicionó formalmente a la FTP (Zancajo y Valiente, 2019b). Por ejemplo, las discusiones sobre la ley de aseguramiento de la calidad establecieron estándares basados en la enseñanza general y academicista para todos los establecimientos (Arellano y Donoso, 2020).

La reactivación del debate sucedió en 2007 tras la publicación de la Estrategia nacional de innovación para la competitividad. El documento, siguiendo los debates que se había tomado la agenda económica, reiteró la urgencia de crear valor en el mercado sofisticando la estructura productiva y agregando conocimiento a la producción a través de un círculo virtuoso de tres pilares: innovación empresarial, ciencia con orientación estratégica y capital humano de calidad, especialmente en sectores productivos con alto potencial competitivo (CNIC, 2007). Aunque al discutir sobre el capital humano el foco estuvo en la educación superior, sus ideas promovieron una transformación de todo la FTP (Zancajo y Valiente, 2019b) y sirvió para la publicación, desde el Ministerio de Educación, de las Bases para una política de formación técnico-profesional en 2009 (MINEDUC, 2009), cuyo diagnóstico presentaba las dificultades que impedían a la EMTP cumplir adecuadamente su propósito y satisfacer las demandas de jóvenes, familias y empresas (MINEDUC, 2009).

Considerando toda la formación técnico-profesional, el documento definió seis principios que debían orientar la discusión de futuras políticas y cuatro áreas de trabajo. Los principios incluyeron la preocupación por la productividad del país; el aprendizaje a lo largo de toda la vida; el desarrollo de competencias técnicas y profesionales; la equidad del acceso y calidad de la oferta educativa; y, finalmente, el acceso a la información relevante, válida y confiable para asegurar la decisión sobre trayectorias, desarrollo de carrera e inversión educativa 
(MINEDUC, 2009). Las áreas de trabajo fueron: institucionalidad; currículum; calidad y pertinencia; y financiamiento.

La preocupación por la institucionalidad busca organizar un sistema de formación que facilite trayectorias progresivas entre niveles y modalidades al conectarlos institucional y curricularmente, un sistema orientado a fortalecer la contribución de la EMTP al desarrollo productivo y la empleabilidad. Las propuestas consideran un marco de calificaciones en los sectores económicos establecidos por la Estrategia nacional de innovación para la competitividad, movimiento que implicaría actualizar la oferta de programas y los perfiles de egreso. Además, implicaba revisar el sistema de títulos y reconocer los aprendizajes y certificación de competencias profesionales (MINEDUC, 2009b).

La segunda área atañe exclusivamente a la EMTP: currículo y aprendizaje. En vínculo con la reforma de 1998, la agenda gubernamental reconoce los problemas de calidad y pertinencia producto, explica el Ministerio de Educación (2009b), de la ausencia de evaluaciones sistemáticas sobre sus avances y eficacia. Las propuestas son tres: reformular el currículo para rediseñar el vínculo con la empresa y dar mayor autonomía a los establecimientos para diversificar su oferta; modificar la evaluación de los aprendizajes y crear capacidades en los establecimientos para evaluar sus perfiles de egreso y definir planes de mejoramiento; y responder a la cuestión docente definiendo estándares disciplinarios y pedagógicos e instrumentos de evaluación. Asimismo, se propuso revisar las normas del ejercicio de la función de aula para facilitar la misma a técnicos de nivel superior y fortalecer la formación continua con foco en el ámbito pedagógico y la actualización de competencias a los cambios tecnológicos.

La preocupación por la calidad y pertinencia de la oferta educativa se impregna de las discusiones generales sobre la urgencia de vincular educacióntrabajo y puede organizarse en cuatro dimensiones: aseguramiento de calidad, financiamiento, actores claves e información.

Respecto al aseguramiento de calidad se plantea la creación de un sistema que permita acreditar instituciones y facilitarles apoyo para desarrollar sus capacidades. El sistema debía permitir invertir en gestión, docencia, equipamiento, 
nivelación, evaluación e innovaciones pedagógicas y curriculares; estar orientado a estándares y al cumplimiento de planes de mejoramiento; y basarse en experiencias de articulación para lograr economías de escala. Estos cambios implicaban repensar el financiamiento de la EMTP, pues la subvención por asistencia era insuficiente. Además, hay medidas para docentes y equipos directivos. A favor de los primeros se propuso un centro para sistematizar y transmitir buenas prácticas; mientras los segundos recibirían un programa de gestión y liderazgo. En relación a la información, se especifican dos formatos. Por un lado, la creación de un observatorio permanente sobre oferta y demanda de capital humano, al menos en tres sectores claves de la economía y donde puedan converger Estado, empresa e instituciones formadoras. Por el otro lado, se definió un sistema de información sobre características, calidad y pertinencia de la oferta y otro sistema de orientación vocacional (MINEDUC, 2009).

El último punto, financiamiento para la demanda, aplica exclusivamente a la educación superior y la formación en el lugar de trabajo (MINEDUC, 2009). Sin embargo, como aparece mencionado en el párrafo anterior, la propuesta contempló modificaciones al financiamiento de la EMTP.

A pesar de sentar un precedente importante en la agenda institucional en el nuevo milenio, las iniciativas definidas en las Bases para una política de formación técnico-profesional en Chile no tuvieron un impacto importante, explican Zancajo y Valiente (2019b). Las propuestas llegaron al final del gobierno y el siguiente debió enfrentar inestabilidad política al reiniciarse un nuevo ciclo de movilizaciones estudiantiles. Las únicas recomendaciones que perduraron descienden de la primera y segunda área de trabajo con la creación de una secretaria ejecutiva técnico-profesional, dentro del Ministerio de Educación, para reorganizar el sistema de FTP, cuyo trabajo más importante fue la actualización curricular en 2013, donde se redujeron las especialidades, favoreciendo las más demandadas y con mejores salarios, se creó el sector económico TIC, se incorporaron habilidades blandas y se dio mayor flexibilidad, al aumentar las horas de libre disposición, explica Weinstein (2013). 
Junto al del nuevo currículo, la secretaría ejecutiva comenzó a diseñar, en conjunto con la UNESCO, un nuevo documento que pudiera traducir las ideas congregadas en las Bases para una política de formación técnico-profesional en una política pública. El trabajo, realizado entre 2014-2015, inició con un diálogo consultivo con las comunidades educativas, sector productivo, academia y ciudadanía y concluyó con la publicación en 2016 de la Política nacional de formación técnico-profesional, la cual debía actuar como hoja de ruta para las tareas a desarrollar durante el segundo gobierno de Michelle Bachelet, mandato que se extendió entre el 11 de marzo de 2014 al 11 de marzo del 2018.

Tal como su predecesor, este documento presenta cuatro áreas de trabajo: calidad, competitividad, trayectorias laborales y educativas articuladas e institucionalidad (MINEDUC, 2016). Empero, aunque algunas ideas se reiteran, otras cambian.

Los debates sobre la calidad se circunscriben principalmente al fortalecimiento del sistema de aseguramiento, ya vigente en el país, buscando perfilar las especificidades de la FTP. Este proceso requería modificar los estándares de formación inicial docente, la evaluación de los establecimientos y los instrumentos de evaluación de estudiantes y docentes. Además, se proponía ejecutar programas de formación continua y otorgar financiamiento para estudios en el extranjero para los docentes. Secundariamente está la modernización del currículo y, por último, la definición de orientaciones para el equipamiento de los establecimientos (MINEDUC, 2016).

El área de competitividad, emprendimiento e innovación se focaliza, tal como su nombre lo indica, en la estrategia de desarrollo y define como herramienta fundamental el marco nacional de calificaciones, el cual debía articular educación-trabajo/FPC-I-FPC-C y dar respuesta a las asimetrías de información entre estudiantes, familias, empleadores y oferentes educativos (MINEDUC, 2016).

El pilar de trayectorias laborales y educativas releva el doble objetivo de la EMTP: dotar de competencias para el empleo y estimular, renovar, diversificar y consolidar competencias a lo largo de toda la vida, vinculando educación media y 
superior TP (ESTP). De esa manera, las propuestas consideraron: el diseño e implementación de un sistema de admisión a la ESTP; aumentar el número de establecimientos TP participantes de un programa de acompañamiento a estudiantes destacados para su ingreso a la ES; fortalecer la articulación entre ambos niveles; y entregar becas y créditos. En todos los casos la mirada está puesta en la reducción de las barreras de acceso y fortalecer la enseñanza superior, particularmente la ESTP.

Finalmente, el área de institucionalidad busca aunar los esfuerzos del Ministerio de Educación, Ministerio de Economía y Trabajo, empleadores, trabajadores e instituciones de formación. En lo concreto y pensando en la EMTP, más que proponer un sistema de formación general, como lo hizo las Bases para una política de formación técnico-profesional, el documento propone la creación de instituciones específicas: un Consejo asesor de formación técnico-profesional, apoyado por consejos regionales. El primero debería actuar como órgano asesor de la presidencia, estar presidido por el Ministerio de Educación y proponer una estrategia nacional multisectorial a renovar cada 5 años. Los consejos regionales apoyarían la contextualización de la estrategia a cada territorio. Asimismo, se propone crear dos instituciones para cada nivel educativo en el MINEDUC: en la EMTP se habla de una unidad de educación media técnico-profesional dentro de la división de educación general. Finalmente, se propone articular los centros de formación técnica estatales a los establecimientos de EMTP y crear consejos locales de educación y trabajo como apoyo a los servicios locales de educación.

Nuevamente hubo problemas en la implementación de las medidas. El marco de calificaciones, por ejemplo, quedó inconcluso y entrampado en discusiones sobre cuál debía ser la institución líder del proceso (Zancajo y Valiente, 2019a). No obstante, hubo dos que pudieron materializarse. La primera, gracias a la modificación de los instrumentos de evaluación docente, permitió el ingreso de los profesores de establecimientos públicos al sistema de carrera docente, el cual reconoce y valora sus trayectorias, habilidades y conocimientos y las traduce en mejoras salariales. La segunda fue la constitución, a partir del Decreto 238, del Consejo asesor de formación técnico-profesional para elaborar 
una Estrategia nacional de formación técnico-profesional 2018-2030 y asesorar al Presidente/a de la República en el análisis y definición de políticas y programas para fortalecer la FTP.

La Estrategia nacional de formación técnico-profesional 2018-2030 vino a establecer la agenda en FTP y fijar una hoja de ruta en el corto, mediano y largo plazo. Tal como la Política nacional de formación técnico-profesional se sostuvo en múltiples debates entre los miembros de los consejos regionales formados, expertos y tomadores de decisión. La discusión giró en torno a tres hitos fundamentales y cinco dimensiones de trabajo. Los hitos incluyeron el marco de calificaciones como instrumento guía, la creación de una institución que coordine el sistema, al menos, durante 5 años y la implementación de un nuevo currículo. En cambio, las cinco propuestas se organizan en tres centrales para la configuración del sistema y dos últimas de soporte: gobernanza e institucionalidad; pertinencia y calidad; trayectorias y rutas formativas; financiamiento; e información y comunicaciones (MINEDUC, 2018a).

La gobernanza e institucionalidad retoma la preocupación por la constitución de un sistema de FTP presente en los dos documentos anteriores. El diagnóstico seguía vigente y urgía crear una agencia de formación técnico profesional, responsable de liderar el sistema de FTP y fortalecer el sistema de aseguramiento de la calidad y la articulación de las rutas formativas (MINEDUC, 2018a).

El área de pertinencia y calidad buscan responder al currículo y las demandas de estudiantes, trabajadores, empleadores y sociedad civil a través de mayor empleabilidad, ingresos, competitividad y productividad. Las propuestas implican, en primer lugar, el uso del marco de calificaciones para orientar la oferta de formación, resultados y procesos de aseguramiento. Ello implicaba alinear y actualizar el currículo de la EMTP y la orientación de la FPC-C. Asimismo, se propuso modificar el sistema de aseguramiento de la calidad definiendo estándares, criterios de evaluación, protocolos de visitas e instrumentos de evaluación de los aprendizajes, así como implementar procesos de acreditación para los establecimientos TP. En tercer lugar, había que reformular la EMTP como primer paso en las trayectorias laborales y educativas, consecuentemente, debía 
revisarse su currículo e implementar apoyos técnico-pedagógico a los liceos. La redefinición curricular debía incluir el marco de calificaciones y articularse con la formación general, las habilidades del siglo XXI, la perspectiva de género y el apoyo a personas con necesidades educativas especiales. La actualización debía ser cada 5 años y tener una revisión profunda cada 10 años. Finalmente, está la preocupación por los docentes, particularmente incentivar la oferta de programas de formación inicial, pensando en quienes ejercen en el nivel sin tener el título en pedagogía, y crear espacios de formación continua en los sectores productivos (MINEDUC, 2018a).

La priorización de las trayectorias y rutas formativas, continúa el Ministerio de Educación (2018a), relevan la conexión con el mercado laboral y la formación general. Así, sus propuestas cubren cinco áreas, de las cuales sólo una atañe especialmente a la EMTP: fortalecer las trayectorias en la educación pública ofreciendo acompañamiento técnico-pedagógico especializado, revisando la oferta de programas regionalmente, generando vínculos con la ESTP estatal, apoyando procesos de certificación de calidad de los establecimientos, fortaleciendo las prácticas de los estudiantes y su formación en alternancia, incentivando las competencias docentes, a través de pasantías en el mundo laboral, e incorporando la evaluación de competencias laborales al egreso de los estudiantes. De las siguientes, hay dos relacionadas indirectamente a la EMTP: crear un sistema de orientación vocacional y laboral que permita tomar decisiones informadas y establecer orientaciones para el reconocimiento de aprendizajes, credenciales y certificaciones en los diferentes niveles y espacios formativos en línea con el marco de calificaciones.

Finalmente, está el financiamiento e información y comunicación, consideradas áreas de soporte. En la primera, las propuestas excluyen completamente a la EMTP al focalizarse en la ESTP. En la segunda, se discute el desarrollo de un sistema de monitoreo de la implementación e impacto de la Estrategia nacional de formación técnico-profesional, tomando como insumo el sistema informacional que se propone construir para la orientación vocacional y laboral. Además, se propone desarrollar investigación sobre la FTP y generar una 
campaña público-privada de valoración social de la modalidad, pues se entiende que la FTP requiere de cambios culturales profundos que escapan a los cambios propuestos (MINEDUC, 2018a).

\section{La construcción social de la EMTP como problema público}

La definición de un problema público no es sencilla: producto del debate colectivo hay transformación y conflicto para determinar la versión oficial (Aguilar, 1993; Dery, 2000; Weiss, 1989). En este camino, es común encontrar elementos que se fortalecen, mientras otros desaparecen. Es decir, continuidades y rupturas que permiten comprender la articulación de la agenda formal a lo largo del tiempo.

\subsection{Las continuidades en el problema público de la EMTP}

Las continuidades en la agenda institucional de la EMTP aparecen en tres temas que cruzan transversalmente los tres documentos, evidenciando su prioridad: vínculo educación-trabajo, calidad educativa e institucionalidad.

Evidentemente, los tres documentos recogen el valor de la FTP en la transformación de la estructura productiva del país. Este punto va más allá de la definición de la EMTP y apunta, tal como lo declararon las Bases para una política de formación técnico-profesional, como uno de los principios, el primero de los seis, que debe orientar el diseño de las políticas públicas en el largo plazo. Se presenta, así, una alineación directa y explícita al marco político que instauró la aparición de la ETP en la agenda gubernamental durante el nuevo siglo y la línea general de la reforma educacional de 1998, la cual buscaba, recuerda Castro y Orellana (2010), responder a las estructuras laborales post-fordistas y adecuarse a los cambios tecnológicos del nuevo siglo.

En el binomio educación-trabajo se articulan dos subtemas. Primero, la preocupación por la abolición del concepto de educación terminal para referirse a la EMTP y, consecuentemente, el interés por potenciar las trayectorias educativas y laborales. En las Bases para una política de formación técnico-profesional estas ideas corresponden a principios para orientar el diseño de las políticas públicas 
que atañen al sector. En los dos documentos oficiales siguientes, adquirían la forma de áreas de trabajo. En este camino, el concepto evoluciona y, aunque su foco de acciones pasa desde la transición EMTP-ESTP al fortalecimiento de la EMTP, la línea base sigue constante. Es importante señalar que esta preocupación no es nueva en la agenda, pues estuvo presente en la reforma educacional de 1965 que permitió, por primera vez en la historia de la FTP en el país, la continuidad legal de estudios superiores para sus egresados (Arellano y Donoso, 2020; Miranda, 2003). Sin embargo, ha tendido a perdurar en el discurso social (Arnold y Krammenschneider, 1999; MINEDUC, 2018a; Sevilla, 2014), incluso cuando la realidad muestra que una alta proporción de egresados ingresa a las universidades e institutos profesionales, a pesar de que lo logran con menores puntajes en las pruebas de selección (Farías y Carrasco, 2012; Orellana, 2018), menores tasas de matrícula e ingreso diferido (Arroyo, Espinoza y Reyes, 2018).

Un segundo subtema, en el marco de la educación-trabajo, es el interés por las competencias técnicas y profesionales. Nuevamente, en el primer documento oficial analizado la idea es un principio que debe impregnar el diseño de la política pública, pero en este caso también es una herramienta concreta. Por un lado, está el marco de calificaciones, el cual refleja una iniciativa para responder al vínculo entre educación-trabajo y, sobre todo, la integración entre la FPC-I y FPC-C. Por el otro lado, está el enfoque de competencias, aunque no es exclusivo de la EFTP, se constituyó como pilar de la reforma curricular de 1998 para destacar el conocimiento, actitudes, habilidades y tareas relevantes para desempeñarse en situaciones laborales específicas (Contreras, 2005; Miranda, 2005).

El enfoque de competencias se grafica en un currículo, cuyo énfasis también es transversal en los tres documentos. Las Bases para una política de formación técnico-profesional lo destaca como vínculo con la empresa e insta a actualizarlo. La misma secretaria técnica técnico-profesional lo hizo y publicó en 2013. La idea persiste en la Política nacional de formación técnico-profesional bajo un nuevo argumento: el currículo debe estar en permanente actualización para sumarse al ritmo de cambio de un mercado en permanente transformación y estar en conversación con el marco de calificaciones. Un interés de renovación y 
vínculación que está alineado con la literatura (Miranda, 2005; Weinstein, 2013; Weller, Gontero y Campbell, 2019) y que seguirán haciendo eco en la agenda institucional al emerger en la Estrategia nacional de formación técnico-profesional, desde donde se suman nuevas preocupaciones a las cuales debe responder el currículo: las habilidades del siglo XXI, la perspectiva de género, el vínculo con la formación general y el apoyo a personas con necesidades educativas especiales. Así, tiene la peculiaridad que, como continuidad, tiende a expandirse.

Junto al vínculo educación-trabajo, el problema público de la EMTP se ha caracterizado por su constante preocupación por la calidad educativa, transformándose tempranamente en uno de los principios que, según las Bases para una política de formación técnico-profesional, debía orientar la agenda. Esta preocupación responde a las demandas ciudadanas contraídas bajo la implementación del modelo neoliberal en educación: la autonomía otorgada a los establecimientos en los 80 s produjo un incremento exagerado e inorgánico de especialidades, planes y programas poco innovadores, ausencia de competencias de empleabilidad $y$, debido a la disminución del financiamiento público, baja adecuación a las necesidades regionales, sectoriales y retraso tecnológico (Castro y Orellana, 2010; Miranda, 2003; Sepúlveda, 2019). Un diagnóstico que no era exclusivo de la EMTP, pues la masificación, privatización, desregulación y falta de políticas de mejoramiento crearon una crisis general en toda la educación media (Bellei et al., 2020). Sin embargo, en esta modalidad afectaban uno de sus propósitos, especialmente crítico considerando que la mayoría de sus estudiantes pertenecía a los estratos de menor nivel socioeconómico: habían bajas tasas de empleo, salario e ingreso para sus egresados (Miranda, 2003).

El cuarto elemento de continuidad en la agenda es la institucionalidad. Un punto que, además, va adquiriendo mayor coherencia y fortaleza de un documento al otro: desde la formación general de un sistema unificado apoyando en la construcción de un marco de calificaciones a la creación de instituciones específicas, como el Consejo asesor de formación técnico-profesional señalado por la Política nacional de formación técnico-profesional y una agencia de formación técnico-profesional en la Estrategia nacional de formación técnico-profesional. Este 
movimiento da a la institucionalidad una presencia más orgánica, fuerte y vinculada a la estructura gubernamental.

\subsection{Las rupturas en el problema público de la EMTP}

Si bien los tres documentos siguen una cadena progresiva, desde el diseño de una política hasta la conformación de una política y estrategia, y fueron discutidos bajo una misma presidencia en el poder ejecutivo de Chile, aunque en dos mandatos distintos, presentan varias rupturas, las cuales se visibilizan en elementos que pierden prioridad en el desarrollo de la agenda institucional. A diferencia de las continuidades, varias de ellas establecidas originalmente como principios en las Bases para una política de formación técnico-profesional, las rupturas parecen ser de tres tipos: de un principio, en los contenidos de las propuestas elaboradas $\mathrm{y}$, probablemente la más interesante, en el enfoque teórico de base que perfila lo que es la FTP.

La primera ruptura sucede a nivel de la información. En las Bases para una Política de formación técnico-profesional la información fue considerada uno de los principios a orientar la discusión, en el documento siguiente desaparece para volver a emergen en la Estrategia nacional de formación técnico-profesional como dimensión de soporte. Entre estos extremos, ha sumado nuevas aristas. Originalmente la discusión sobre la información aparece limitada como principio al estudiante y su familia: el Estado debía asegurarles información relevante, válida y confiable para que decidieran sus trayectorias. Como propuesta, en el apartado de calidad, suma el diseño de un sistema sobre la oferta. Posteriormente, en el último de los documentos revisados, se suman tres aristas: como sistema de monitoreo de la estrategia en discusión, como incentivo a la investigación y como campaña de valoración social. Se recoge, de esa manera, una respuesta más integral que no sólo satisface a los estudiantes, trabajadores y sus familias, sino también al aparato estatal y la sociedad.

El segundo tipo de ruptura se observa en las propuestas discutidas, por ejemplo, sobre los docentes y el financiamiento. En ambos casos, y a diferencia de 
lo que sucede con la ruptura respecto al princio de la información, la agenda se contrae al hablar de los profesores y el financiamiento de la EMTP.

La prioridad dada a los docentes, tal como sucede con la calidad educativa, se nutre también de las discusiones que bordearon la agenda y que culminaron en la reforma educacional de 1998, entre ellos, recuerda Arellano (2001) está la formación inicial y continua. Empero, aunque presenta cierta continuidad en los tres documentos revisados, no sucede lo mismo con alguna de sus propuestas. En el primer documento discutido, la preocupación por la cuestión docente es amplia: primero, en el pilar de currículum, aparecen propuestas para diseñar estándares de formación inicial e instrumentos de evaluación, revisar las normas que regulan el ejercicio de aula y fortalecer la formación; luego, en el pilar de calidad, se habla de construir centros de apoyo para sistematizar y transmitir buenas prácticas pedagógicas. De estas cinco propuestas, la tercera y la última desaparecen en la Política nacional de formación técnico-profesional y no vuelven a la agenda. Entre ambas, se extraña sustancialmente la tercera. El Decreto 352, publicado en 2003, reglamenta el ejercicio de la función docente y permite que, ante ausencia de docentes titulados de pedagogía, puedan impartir clases de la formación diferenciada profesionales o técnicos de áreas afines, incluyendo a egresados de la EMTP. Si bien ello permitió responder al potencial déficit docente producido por el cierre de la carrera de enseñanza media técnico-profesional en las universidades chilenas en los 80 s por ausencia de demanda y oferta (MINEDUC, 2012), trajo consigo otro. Como señalan Barrientos y Navío (2015), la enseñanza requiere habilidades y actitudes específicas que la especialización técnico profesional no necesariamente ofrece a sus egresados. El enfoque de competencias laborales, continúa Cid et al. (2017), impone grandes desafíos a los profesores, quienes deben promover y gestionar el rediseño curricular, ejecutar ajustes didácticos, re-evaluar el perfil de egreso y desarrollar competencias genéricas y específicas. El déficit de profesionales titulados de carrera de pedagogía y la mayor cantidad de titulados de otras áreas ha llamado la atención de la literatura especialidad. Por ejemplo, se ha propuesto modificar dichas normas para pedir como requisito la experiencia laboral relevante (CPP-UC, 2018). 
Respecto al financiamiento, la contracción es más extrema al desaparecer del debate el rediseño de la subvención escolar propuesto en el primer documento, una subvención fija que el Estado entrega por la asistencia mensual del estudiante al establecimiento educativo. Como se observó en la Política nacional de formación técnico-profesional, el financiamiento en EMTP queda reducido a la definición de orientaciones para el equipamiento de los establecimientos y, en la Estrategia nacional de formación técnico-profesional pasa a concentrarse en la ESTP y capacitación a trabajadores. Ahora bien, este cambio, aunque insatisfactorio con la EMTP, ha ayudado a reforzar la ESTP y es concordante con la evidencia: las precarias condiciones económicas de los estudiantes de la EMTP explican su ingreso diferido a la educación superior (Larrañaga, Cabezas y Dussaillant, 2013) y, en caso de entrar, su compatibilización con el trabajo (Sepúlveda y Valdebenito, 2014). Sin embargo, deja en el aire una demanda fuertemente sentida por las comunidades educativas de la EMTP.

Por último, es importante detenerse en la ruptura del enfoque teórico base. En los tres documentos revisados aparecen elementos propios del enfoque de capital humano. Una perspectiva ampliamente difundida por organismos internacionales como el Banco Mundial y la OIT, recuerda Sevilla (2014) y Zancajo y Valiente (2019a). Además del valor otorgado al mercado laboral, este enfoque prioriza el diseño de marcos nacionales de calificaciones, la formación basada en competencias y la política de rendición de cuentas. Cada uno de estos elementos propios del enfoque de capital humano están presentes en los tres documentos oficiales revisados, así como demandas que buscan reformularlo.

Las rupturas de enfoque modifican la definición misma de FTP, pues ya no se trata de conciliar las trayectorias educativas y laborales únicamente con las necesidades de desarrollo del país, como lo hizo las Bases para una política de formación técnico-profesional, sino también con las expectativas y capacidades de sus estudiantes y trabajadores. El desarrollo económico también recoge un nuevo adjetivo: la sustentabilidad. Estas ideas son propias del enfoque de derecho social, señalan Arellano y Donoso (2020) y Zancajo y Valiente (2019a). 
Un enfoque que, además, ilumina el entendimiento sobre la estructura de oportunidades que marca diferencias en las trayectorias de estudiantes y trabajadores, pues entiende que sus decisiones sobre seguir estudiando, trabajar o permanecer inactivos dependen de distinciones de clase, género, raza y discapacidad. Desigualdades que urge intervenir, especialmente aquellas que perjudican el egreso de la enseñanza media (Zancajo y Valiente, 2019a). Una tarea que la literatura insistía en destacar desde hace años: los egresados de establecimientos TP presentan menores puntajes en las pruebas de selección universitaria que los egresados de la EMCH (Farías y Carrasco, 2012; Larragaña, Cabezas y Dussaillant, 2013). Un fenómeno que también les ofrece menos oportunidades para estudiar en instituciones o carreras de más prestigio. Así, las propuestas comienzan a aparecer en el segundo documento y se busca, por ejemplo, implementar un sistema de admisión a la educación superior diferenciado (MINEDUC, 2016) o fortalecer los establecimientos técnicos profesionales a través del apoyo pedagógico (MINEDUC, 2018a).

La inclusión de nuevas temáticas en el currículo también muestra la brecha entre ambos enfoques, pues esta expansión temática no es propia del enfoque de capital humano (Sevilla, 2017) y sólo pueden emerger ante su ruptura. Un camino que se sincroniza con los debates internacionales más vinculados al enfoque de derechos sociales. No hay que olvidar, por ejemplo, que la UNESCO (2015) reitera la preocupación por el medio ambiente y la igualdad de oportunidades de aprendizaje y de género en la agenda de la FTP.

En un escenario de conciencia y responsabilidad social, y a diferencia del enfoque de capital humano, el enfoque de derechos también acusa la ingenuidad de creer que el mercado puede regularse a sí mismo y encontrar un equilibrio entre demanda y oferta (Valiente, Zancajo y Jacovkis, 2020). Por el contrario, incentiva la participación del Estado en la provisión y regulación de la oferta educativa (Zancajo y Valiente, 2019a). Esta ruptura es particularmente evidente en la Estrategia nacional de formación técnico-profesional, donde el rol del Estado se amplía desde la rendición de cuentas a la toma de decisiones estratégicas, normativas y regulatorias. El Estado, a través del fortalecimiento de la institucionalidad, pasa a 
tener la responsabilidad de ser un ente regulador del sistema de formación y debe liderar los diálogos y contextualizar las definiciones de la agenda a las necesidades de cada sector productivo y territorio, proceso en que debe acompañar a las instituciones formadoras para la implementación de las medidas que les atañen. En contrapartida cada sector productivo debe entrar en este diálogo informando sus necesidades actuales y futuras, pues ello permitirá definir el currículo y los distintos programas formativos en pos de cumplir con calidad y pertinencia.

Consecuente con este enfoque, y a diferencia de la elaboración del primer documento donde sólo participaron expertos, el debate reflejado en la construcción de los dos documentos siguientes consideró la participación de los principales actores involucrados, destacando entre ellos los docentes.

\section{Conclusiones}

La agenda institucional en la enseñanza media técnico-profesional en Chile no ha estado quieta ni silenciada como se puede observar en el primer apartado discutido, después de la introducción. Por el contrario, los debates entre expertos, tomadores de decisión, empleadores, formadores y los estudiantes y sus familias se ha traducido en tres documentos oficiales que contienen varias continuidades, destacando la calidad educativa y la institucionalidad, y rupturas, entre ellas la cuestión docente y el financiamiento. Continuidades y rupturas desde donde se han levantado múltiples propuestas y desafíos de cambio que buscan, al final de cuentas, mejorar la formación técnico-profesional, en general, y la EMTP, en específico. A pesar de este auge, la mayoría de las propuestas discutidas en las Bases para una política de formación técnico-profesional en Chile (MINEDUC, 2009), la Política nacional de formación técnico-profesional (MINEDUC, 2016) y la Estrategia nacional de formación técnico-profesional (MINEDUC, 2018a) no han logrado concretizarse aún y transformar significativamente la educación que reciben los estudiantes que ingresan a la enseñanza media técnico-profesional en Chile. Excepto por algunas de las propuestas, mencionadas anteriormente, lo cierto es que la FTP ha estado fuera de las discusiones educativas más amplias que enfrenta el país. Este problema no es exclusivo de Chile y, autores como 
Palmer (2014), lo explican en la mayor atención que recibe y atrae la formación general. Gracias a ello, la formación general en Chile, conocida como enseñanza media científico-humanista, ha concentrado toda la atención en temas como la reformulación de las pruebas estandarizadas que deben rendir todos los estudiantes para, por un lado, conocer y diagnosticar la calidad de enseñanza en el país y, por otro lado, permitir el ingreso a la educación superior (Arellano y Donoso, 2020; MINEDUC, 2012; UNESCO, 2015). Un modelo de enseñanza general que predomina incluso entre los docentes técnicos-profesionales y que ha dificultado la implementación exitosa del enfoque de competencias impulsado en la reforma curricular de 1998. Bien lo explica Díaz-Barriga (2012), la lógica imperante sigue siendo el recorte uni-disciplinar y enciclopedista que prima la enseñanza de temas y abundantes contenidos, así como la estructuración en asignaturas más bien insulares, aunque hoy se llamen módulos.

En este camino, no todo está perdido $y$, en realidad, hay victorias significativas a la vista. Las Bases para una política de formación técnicoprofesional en Chile, como primer documento oficial publicado en el nuevo milenio, estableció tempranamente las ideas centrales de la agenda institucional en FTP, al tiempo que, otra parte de su contenido se ha modificado, la mayoría de forma positiva y expansiva. La ruptura paradigmática es probablemente la más provechosa de las discontinuidades, sin obviar los cambios instaurados en otras áreas. Una ruptura de enfoque que ha permitido, entre otras cosas, exigir mayor participación del Estado en la provisión y regulación de la oferta educativa para la EMTP (Zancajo y Valiente, 2019a). Como bien se mostró en la descripción de la Estrategia nacional de formación técnico-profesional (MINEDUC, 2018a), hoy el Estado tiene la responsabilidad de regular la educación, pedir cuentas y acompañar a las instituciones formadoras.

Es interesante notar que este cambio de paradigma fue impulsado principalmente por los movimientos estudiantiles que se generaron en Chile entre 2011-2013, movimientos sociales a los cuales la Política nacional de formación técnico-profesional hace eco en su introducción y, al hacerlo, parece extenderlos hacia el resto de la agenda gubernamental en FTP. De esa forma, si bien los 
mismos movimientos estudiantiles llegaron a entorpecer la puesta en marcha de las recomendaciones elaboradas en 2009 al introducir demandas más propias de la formación general, lo cierto es que también introdujeron ideas valiosas en la agenda formal de la FTP, recuerda Zancajo y Valiente (2019b). Este diálogo con los movimientos sociales evidencia las fructíferas conexiones que se producen entre la agenda institucional y la agenda sistémica, la cual corresponde a los temas que son objeto de atención y/o conocimiento por parte de una comunidad, quienes consideran que su solución requiere algún tipo de intervención estatal (Elder y Cobb, 1993). Un camino de diálogo que no es sencillo ni lineal, pero que representa la tarea política de los líderes, organizaciones y partidos políticos para recoger las necesidades y demandas ciudadanas y llevarlas al debate político en busca de solución (Aguilar, 1993).

Ahora bien, aunque los cambios introducidos en respuesta a las necesidades y demandas ciudadanas han modificado sustancial y positivamente la agenda institucional de la FTP en Chile, hay algunas transformaciones que aún deben profundizarse. Aquí, se discuten al menos dos.

La primera de ellas es la innovación educativa. La educación técnicoprofesional está cubierta de múltiples oportunidades para innovar al transformar los paradigmas tradicionales de enseñanza, el currículo, las evaluaciones y las relaciones con los estudiantes, siguiendo la definición de la misma dada por autores como Rivas (2017), pero tiende a seguir estancada en la matriz tradicional de enseñanza. El enfoque de competencias, la formación en alternancia, las habilidades del siglo XXI y las nuevas tecnologías son sólo algunos de los ejemplos que podrían representar herramientas para la innovación educativa, nos recuerda un informe emitido por el Ministerio de Educación de Chile (2018b) acerca específicamente de la innovación en la EMTP, pero aún no llegan a todos los colegios y a todas las salas de clases; es más, tal vez sólo han aterrizado en algunas de ellas y en contadas asignaturas.

El otro tema que aún requiere profundizarse cuando hablamos de la FTP es la perspectiva de género. Se mencionó antes como esta temática ha entrado a la agenda gubernamental de Chile a través del currículo, empero, sus demandas 
debiesen ser más amplias. La distribución de los estudiantes según su género entre los distintos sectores económicos impartidos por la EMTP, por citar un caso, evidencia gran inequidad: las mujeres predominan en los sectores de servicios personales, los cuales tienden a reportar menores salarios al momento de egresar al mundo laboral (Larragaña, Cabezas y Dussaillant, 2013; MINEDUC, 2012; Sevilla y Ortiz, 2019). Una separación que también afecta la distribución de los profesores que ejercen en la modalidad, como se observa en las bases del MINEDUC (2019): las docentes mujeres que ejercen en la EMTP también tienden a concentrarse en las especializadas asociadas al sector de servicios. Ambas dinámicas reproducen las lógicas de segmentación por género que persisten en el orden social mayor (Sepúlveda, 2017) y que deben comenzar a romperse. Mucho se ha dicho sobre la escasez de mujeres en las ingenierías (OCDE, 2015), pero poco se ha debatido sobre la concentración de mujeres en las especialidades de hotelería, salud y educación y su escasez en contabilidad o mecánica. De esa manera, tanto en innovación educativa como en el enfoque de género debe organizarse una intervención más profunda, de la cual la EMTP no puede quedar ajena.

\section{Referencias bibliográficas}

Aguilar, L. (1993). Estudio introductorio. En L. Aguilar (Ed.), Problemas públicos y agenda de gobierno (pp. 13-74). México D.F.: Miguel Ángel Porrúa.

Arellano, J. P. (2001). La reforma educacional Chile. Revista de la CEPAL, 73, 83-94. Arellano, M. y Donoso, G. (2020). Formación técnico profesional en Chile: Aportes a la transformación de las personas y al desarrollo del país. En M. T. Corvera y G. Muñoz (Ed.), Horizontes y propuestas para transformar el sistema educativo chileno (pp. 336-359). Santiago de Chile: Ediciones Biblioteca del Congreso Nacional de Chile.

Arnold, R. y Krammenschneider, U. (1999). Caught between private and public interests: vocational training in Chile. International Journal of Sociology, 29(2), 5-28. Arroyo, C., Espinoza, S. y Reyes, L. (2018). Trayectorias formativas y laborales de los jóvenes en Chile. Santiago de Chile: Comisión Nacional de Productividad. 
Barrientos, C. y Navío, A. (2015). Formador de la educación superior técnico profesional: percepciones sobre su trabajo docente. Estudios Pedagógicos, 41(1), 45-61.

Bellei, C., Contreras, M., Valenzuela, J. P. y Vanni, X. (2020). Introducción: el liceo en tiempos turbulentos. En C. Bellei, M. Contreras, J. P. Valenzuela y X. Vanni (Ed.), EI liceo en tiempos turbulentos: ¿cómo ha cambiado la educación media chilena? (pp. 9-70). Santiago de Chile: LOM Ediciones y Universidad de Chile, CIAE.

Blumer, H. (1997). Social problems as collective behavior. Social Problems, 18(3), 298-306.

Castro E. y Orellana, W. (2010). La educación media técnico profesional en Chile: entre la desarticulación y la indefinición. Intersecciones Educativas, 2, 73-81.

Centro de Políticas Públicas UC (CPP-UC). (2018). Estado y nudos críticos de la formación técnica en Chile. Santiago de Chile: Centro de Políticas Públicas UC (CPP-UC).

Cid, J., Cuadra, L., Cuevas, S. y Villalobos, A. (2017). Articulación educacióntrabajo: Un estudio desde la mirada de los docentes en la educación técnica regional chilena y sus necesidades de perfeccionamiento pedagógico. Información Tecnológica, 28(1), 25-34.

Consejo Nacional de Innovación para la Competitividad (CNIC). (2007). Estrategia nacional de innovación para la competitividad. Santiago de Chile: Ministerio de Economía.

Contreras, M. (2005). Elaboración del currículo de la enseñanza media técnicoprofesional con enfoque en competencias laborales. Pensamiento Educativo, 36, 294-310.

Dery, D. (2000). Agenda setting and problem definition. Policy Science, 21(1), 37-47.

Díaz-Barriga, F. (2012). Reformas curriculares y cambio sistémico: una articulación ausente pero necesaria para la innovación. Revista Iberoamericana de Educación Superior, 3(7), 23-40.

Elder, C. y Cobb, R. (1993). Formación de la agenda. El caso de la política de ancianos. En L. Aguilar (Ed.), Problemas públicos y agenda de gobierno (pp. 77104). México: Miguel Ángel Porrúa. 
Farías, M. y Carrasco, R. (2012). Diferencias en resultados académicos entre la educación media técnica profesional y humanista científica en Chile. Calidad en la Educación, 36, 88-121.

Larrañaga, O., Cabezas, G. y Dussaillant, F. (2013). Informe completo del estudio de la educación técnico profesional. Santiago de Chile: Programa de las Naciones Unidas (PNUD-Chile).

Llisterri, J., Gligo, N., Homs, O. y Ruíz-Devesa, D. (2014). Educación técnica y formación profesional en América Latina. El reto de la productividad. Caracas: Corporación Andina de Fomento (CAF) y Banco de Desarrollo de América Latina.

MINEDUC (2009). Bases para una Política de Formación Técnico-Profesional en Chile. Santiago de Chile: Ministerio de Educación.

MINEDUC (2012). Educación técnica-profesional en Chile. Antecedentes y claves de diagnóstico. Santiago de Chile: Centro de estudios, Ministerio de Educación. MINEDUC (2016). Política Nacional de Formación Técnico-Profesional. Santiago de Chile: Ministerio de Educación.

MINEDUC (2018a). Estrategia Nacional de Formación Técnico-Profesional. Santiago de Chile: Ministerio de Educación.

MINEDUC (2018b). \#SoyTP. Experiencias innovadoras para el fortalecimiento de la educación técnico profesional. Santiago de Chile: Ministerio de Educación.

MINEDUC (2019). Bases de datos de cargos docentes. Santiago de Chile: Ministerio de Educación.

Miranda, M. (2003). Transformación de la educación media técnico-profesional. En C. Cox. (Ed.), Políticas educacionales en el cambio de siglo. La reforma del sistema escolar de Chile (pp. 375-417). Santiago de Chile: Universitaria.

Miranda, M. (2005). Aprendizaje técnico en un enfoque de competencias laborales. Pensamiento Educativo. Revista de Investigación Educacional Latinoamericana, 36(1), 220-232.

Núñez, M. (2013). El docente en el enfoque por competencias. Pensamiento, Papeles de Filosofía, 1, 177-186.

Orellana, R. (2018). El efecto de la educación media técnico-profesional en la Prueba de Selección Universitaria. ¿Logran los liceos TP con destacado SIMCE replicar su 
éxito en la PSU? Tesis para optar al grado de magíster en políticas públicas, Facultad de Economía y Negocios. Santiago de Chile: Universidad de Chile. OCDE (2015). The ABC of gender in education. Paris: OCDE.

Palmer, R. (2014). Technical and vocational skills post-2015: Avoiding another vague skills goal? International Journal of Educational Development, 39, 32-39.

Rivas, A. (2017). Cambio e innovación educativa. Las cuestiones cruciales. Buenos Aires: Fundación Santillana.

Sepúlveda, L. (2017). La educación técnico profesional en América Latina, Retos y oportunidades para la igualdad de género. Santiago de Chile: CEPAL.

Sepúlveda, L. (2019). Cincuenta años de la Educación Media Técnico Profesional en Chile: Continuidad, rupturas y desafíos para el futuro (a manera de introducción). En L. Sepúlveda y M. Valdebenito (Ed.), Educación Técnico Profesional ¿Hacia dónde vamos? Santiago de Chile: Ediciones Universidad Alberto Hurtado.

Sepúlveda, L. y Valdebenito, M. J. (2014). Aspiraciones y proyectos de futuro de estudiantes de enseñanza técnica-profesional. ¿Es pertinente un sistema diferenciado en la enseñanza media? Polis, 38.

Sevilla, M. P. (2014). La educación técnica en Chile y Estados Unidos desde una perspectiva histórica y comparada. Calidad de la Educación, 40, 298-317.

Sevilla, M. P. (2017). Panorama de la educación técnica profesional en América Latina y el Caribe. Santiago de Chile: CEPAL.

Sevilla, M. P. y Ortiz, I. (2019). Demandas y desafíos de la educación técnicoprofesional secundaria en América Latina. En L. Sepúlveda y M. Valdebenito (Ed.), Educación Técnico Profesional ¿Hacia dónde vamos? (pp. 91-114). Santiago de Chile: Ediciones Universidad Alberto Hurtado.

UNESCO. (2015). Recomendación relativa a la Enseñanza Técnica y Profesional (EFTP). Paris: UNESCO.

Valiente, O., Zancajo, A. y Jacovkis, J. (2020). The coordination of skill supply and demand in the market model of skill formation: testing the assumptions for the case of Chile. International Journal of Lifelong Education, 39(1), 90-103.

Weinstein, A. (2013). Diagnóstico de la educación técnico-profesional: estado de la situación y agenda futura. Santiago de Chile: Libertad y Desarrollo. 
Weiss, J. (1989). The power of problem definition: the case of government paperwork. Policy Science, 22, 97-121.

Weller, J., Gontero, S. y Campbell, S. (2019). Cambio tecnológico y empleo: una perspectiva latinoamericana. Riesgos de la sustitución tecnológica y desafíos de la generación de nuevos puestos de trabajo. Santiago de Chile: CEPAL.

Zancajo, A. y Valiente, O. (2019a). Evolución de las políticas ETP en Chile: entre el capital humano y el derecho a la educación. En L. Sepúlveda y M. Valdebenito (Ed.), Educación Técnico Profesional ¿Hacia dónde vamos? (s.n.p). Santiago de Chile: Ediciones Universidad Alberto Hurtado.

Zancajo, A. y Valiente, O. (2019b) TVET policy reforms in Chile 2006-2018: between human capital and the right to education. Journal of Vocational Education \& Training, 71(4), 579-599.

Fecha de recepción: 2 de septiembre de 2020 Fecha de aceptación: 16 de noviembre de 2020

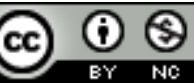

Revista Educación, Política y Sociedad (ISSN 2445-4109) está distribuida bajo una Licencia Creative Commons Atribución-NoComercial 4.0 Internacional 